ISSN electrónico: $1585-5210$

DOI: https://doi.org/10.14201/rmc2021173175177

\title{
REVISTA DE MEDICINA Y CINE: ORIGEN Y EVOLUCIÓN
}

\author{
Journal of Medicine and Movies: Origin and evolution
}

\author{
Enrique GARCÍA SÁNCHEZ \\ Departamento de Ciencias Biomédicas y del Diagnóstico. Facultad de Medicina. Universidad de Salamanca \\ (España). IUCE (Instituto Universitario de Ciencias de la Educación). Universidad de Salamanca (España). \\ IBSAL (Instituto de Investigación Biomédica de Salamanca) (España).
}

Correo electrónico: engarsan@usal.es

Recibido: 6 de febrero de 2021

Aceptado: 7 de abril de 2021

Tendríamos que remontarnos a finales de 1996 para comprender el origen de la Revista de Medicina y Cine (RMC). Por aquel entonces, un profesor de la Universidad de Salamanca (USAL) contactó con el profesor José Elías García Sánchez para intentar paliar el escaso número de Asignaturas de Libre Elección que existían en algunos planes de estudio de titulaciones del ámbito biosanitario. La demanda de los estudiantes era alta ya que debían cursar un elevado número de créditos y la oferta por parte de los profesores de la USAL era limitada. Se ofreció a proponer alguna asignatura que compaginara el estudio de la microbiología / enfermedades infecciosas desde una perspectiva humanística con el fin de complementar la formación de los estudiantes. La primera aproximación fue ofertar una asignatura denominada Videoformación en Microbiología Clínica que se impartió en el curso 1997-98.
A la propuesta del profesor José Elías García Sánchez nos fuimos incorporando otros profesores del ámbito de la Microbiología Médica y la oferta se fue ampliando y diversificando. Así, a esta primera experiencia le siguieron otras más a partir del curso 1998-99, como fueron Las enfermedades infecciosas en el cine, EI SIDA en el cine, La microbiología médica en el cine, La tuberculosis en el cine y otras artes, Microbiología médica y enfermedades infecciosas en el cine y otras artes, Patógenos y enfermedades infecciosas emergentes y reemergentes en el cine y otras artes, Microbiología médica y enfermedades infecciosas en el cine y Medicina, enfermedad y cine, entre otras. El número de estudiantes matriculados en estas asignaturas fue muy elevado en todos los cursos en los que se impartieron y ese fue uno de los motivos por los que la oferta se fue variando a lo largo de los cursos académicos y tuvo el fruto 


\section{REVISTA DE MEDICINA Y CINE: ORIGEN Y EVOLUCIÓN \\ ENRIQUE GARCÍA SÁNCHEZ}

esperado ya que diversificó la formación de los estudiantes, se potenciaron los aspectos humanísticos y culturales, se enseñó a ver la ciencia desde otra perspectiva y todos, incluidos los profesores, fuimos aprendiendo a mejorar nuestras actividades formativas, metodologías docentes y a poner a punto sistemas de evaluación eficaces para medir los avances de los estudiantes.

Estas experiencias fueron el preludio de una propuesta, también promovida por el profesor José Elías García Sánchez en el año 2004, que fue ¿Por qué no hacemos una revista? La respuesta no se hizo esperar y un pequeño, muy pequeño grupo, de profesores nos unimos de forma entusiasta a colaborar para sentar las bases de lo que iba a ser la RMC.

Se establecieron contactos con el Servicio de Publicaciones de la USAL. Los técnicos nos asesoraron sobre procedimientos a seguir. Se planteó, al entonces director de este servicio, esta posibilidad, lo vio con buenos ojos y apoyó la propuesta. En aquellos momentos era importante contar con el apoyo de la USAL y que el ISSN de la revista fuera institucional para dar mayor credibilidad al proyecto.

Se comenzó a trabajar de forma denodada. Es preciso comentar que el apoyo de la USAL ha sido errático a lo largo de los años. Esto ha estado condicionado por el paso de diferentes directores del Servicio de Publicaciones de la USAL. En algunos casos no estuvieron interesados en este proyecto y en otros no alcanzaron a valorar el interés que tenía.

Lo único que se ha mantenido constante a lo largo de estos 17 años ha sido el apoyo de los Técnicos del Servicio que han estado, en todo momento, pendientes de ayudar en todos los aspectos que le ha sido posible y que han intentado darle la mayor visibilidad posible a la RMC. Quiero darle las gracias a Fernando, Angel e Iván por su apoyo incondicional a lo largo de estos casi 17 años.

La RMC inicia su andadura en el año 2005 con un editorial titulado Medicina y cine ¿Por qué ${ }^{1}$ y se cierra este número con un artículo denominado Metodología docente para la utilización del cine en la enseñanza de la microbiología médica y las enfermedades infecciosas ${ }^{2}$. En estas dos publicaciones se sintetiza el ideario de la RMC. Por qué era necesaria esta revista y como hacer viable el proyecto.

Han pasado los años, la RMC se ha consolidado y por fin, en el año 2020, con una nueva dirección en el Servicio de Publicaciones, llegó un momento importante en el devenir de la RMC, fue la inclusión de la revista en Scielo. A partir de ese momento se obtuvo un apoyo imprescindible por parte de la USAL que fue externalizar la maquetación. Procediéndose, en ese momento, a realizar un nuevo cambio de imagen.

Durante todos estos años, la revista se ha mantenido gracias a los autores que han creído en ella y han aportado valiosos artículos de alta calidad, a los revisores anónimos que han realizado una tarea encomiable y a un reducido número de profesores implicados en sacarla adelante con mucho esfuerzo personal que no han tenido apoyo profesional o institucional como ya se comentó en el volumen 7 número 3-4 de 2011 donde se indicaba: "Como a otras actividades la crisis ha afectado a la Revista de Medicina y Cine, aunque sea la única revista bilingüe que edita Ediciones Universidad de Salamanca, y en este momento no puede financiarse si quiere mantenerse en la línea habitual. La única ayuda recibida en todos estos años ha procedido del Vicerrectorado de Investigación de la Universidad de Salamanca, vaya desde aquí una vez más nuestro agradecimiento, y se produjo en 2010. Dado que Ediciones Universidad de Salamanca no está dispuesta a financiar las traducciones ni la gestión informática, a pesar de ser servicios que se pueden dar desde la propia Universidad sin coste adicional, ya que existen servicios específicos que pueden cubrirlo, el Comité Editorial se ve obligado a partir del año 2012 (volumen 8) a publicar los artículos en el idioma que se reciban en castellano o inglés, o en ambos si así son remitidos por los autores. Se intentará mantener 


\section{REVISTA DE MEDICINA Y CINE: ORIGEN Y EVOLUCIÓN \\ ENRIQUE GARCÍA SÁNCHEZ}

los cuatro números, pero puede ocurrir, como ya pasó el año pasado y éste mismo, por motivos económicos, que alguno sea doble. Por último, se suprimirá la versión en ht/m. Estas son las medidas que los editores y el comité de redacción pondrán en marcha para garantizar la supervivencia, en épocas de crisis de una revista, que tiene un sinfín de lectores, un sinfín de suscriptores y un sinfín de citas. ¿Si hasta los animales cuidan de sus hijos, no íbamos a hacer lo mismo los humanos?"3.

Si se revisa el histórico de la RMC se pueden observar diferentes etapas. Bastaría analizar de forma aleatoria algunos artículos de diferentes años para apreciar la evolución en la calidad de la maquetación y los cambios que se han ido introduciendo.

Por otra parte, es necesario dejar constancia de algunos hitos relevantes como ha sido la apertura de la revista a aspectos relacionados con la literatura y la salud que fue anunciado en el volumen $10(4)$ de $2014^{4}$ y que en el volumen 11(1) de 2015 se publicó el primer artículo en forma de un editorial ${ }^{5}$. Posteriormente se han incorporado otros aspectos artísticos como la pintura ${ }^{6}$ o el comic ${ }^{7}$ entre otros, pero siempre siendo fieles a las directrices fundacionales "Revista de Medicina y Cine / Journal of Medicine and Movies es una publicación que analiza los contenidos biosanitarios ... con fines educativos, discursivos, de mentalización y divulgación".

Gracias a Elías por sus buenas ideas, por su trabajo y dedicación que ha hecho posible que estos sueños se hayan hecho realidad.

\section{Referencias}

1. García Sánchez JE, Trujillano Martín I, García Sánchez E. Medicina y cine ¿Por qué? Rev Med Cine. 2005;1(1):1-2.

2. Fresnadillo Martínez MJ, Diego Amado C, García Sánchez E, García Sánchez JE. Metodología docente para la utilización del cine en la enseñanza de la microbiología médica y las enfermedades infecciosas. Rev Med Cine. 2005;1(1):17-23.

3. García Sánchez JE, García Sánchez E. Crisis medicina y cine. Rev Med Cine. 2011;7(3-4): 83-4.

4. García Sánchez JE, García Sánchez E. Hacia la segunda década de la Revista de Medicina y Cine. Rev Med Cine. 2014;10(4):155-6.

5. Baños JE, Guardiola E. Medicina y Literatura: el valor de las palabras. Rev Med Cine. 2015;11(1):3-4.

6. Hidalgo A, Hidalgo C, Cantabrana B. Contribución del arte a la formación de los estudiantes de medicina. Rev Med Cine. 2018;14(1):53-62.

7. García Sánchez JE, García Sánchez E. La microbiología médica y las enfermedades infecciosas en el cómic. Rev Med Cine. 2020;16(1):53-4. 